\title{
Next-generation anthracycline for the management of small cell lung cancer: focus on amrubicin
}

\author{
Michiko Yamamoto \\ Akira Takakura \\ Noriyuki Masuda \\ Department of Respiratory Medicine, \\ Kitasato University School \\ of Medicine, Kitasato, Japan
}

\begin{abstract}
Amrubicin is a totally synthetic anthracycline anticancer agent that acts as a potent topoisomerase II inhibitor. Amrubicin has been approved in Japan for the treatment of lung cancer, and the results from clinical studies of amrubicin as a single agent or as part of combination regimens for lung cancer, particularly for small cell lung cancer, conducted in Japan and overseas have been promising. Amrubicin should be included among new treatment strategies especially for chemoresistant patients. Here, preclinical, pharmacological, and clinical data on the use of amrubicin for the treatment of small cell lung cancer are reviewed.
\end{abstract}

Keywords: amrubicin, anthracycline, small-cell lung cancer, extensive disease

\section{Introduction}

Small-cell lung cancer (SCLC) accounts for $10 \%$ to $15 \%$ of all primary lung cancer and is a rapidly growing tumor that is highly responsive to chemotherapy and radiotherapy (Sher et al 2008). Nonetheless, after apparently successful induction therapy, most patients experience disease relapse and die within 2 years. Despite the introduction of new chemotherapeutic agents, including irinotecan, topotecan, paclitaxel, and gemcitabine, for the treatment of SCLC in the 1990s, few advances in the prolongation of survival in patients with this disease have been made. To control SCLC more effectively, new drugs are needed that are effective in patients who have not responded to standard treatment and who may have multidrug-resistant tumors. One such new effective agent may be amrubicin; the use of this drug has extensively been examined and approved for the treatment of both SCLC and non-SCLC in Japan.

\section{Amrubicin}

\section{Structure and characteristics}

The anthracyclines tested clinically so far have been limited to those produced by fermentation or semisynthetic processes. In contrast, 9-aminoanthracycline, known as amrubicin, is a fully synthetic drug. Amrubicin differs from daunosamine in that it contains a 9-amino group and a simple sugar moiety (Figure 1) (Ishizumi et al 1987). Amrubicin is converted to its active 13-hydroxy metabolite, amrubicinol, in liver, kidney, and tumor tissues through the reduction of its C-13 ketone group to a hydroxy group by the action of cytoplasmic carbonyl reductase. Other enzymes metabolizing amrubicin and amrubicinol are NADPH-dependent P450 reductase and $\mathrm{NAD}[\mathrm{P}] \mathrm{H}-$ dependent quinone oxidoreductase. Despite the similarity of its chemical structure to that of a representative anthracycline, doxorubicin, amrubicin's mode of action differs from that of doxorubicin. Amrubicin and amrubicinol are inhibitors of DNA topoisomerase II, exerting their cytotoxic effects by stabilizing a topoisomerase II-mediated cleavable complex, and are approximately only one-tenth as potent as doxorubicin in producing DNA intercalation (Hanada et al 1998). 


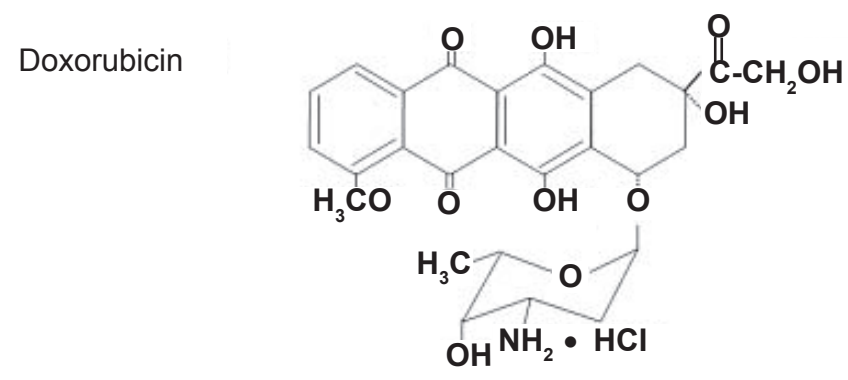

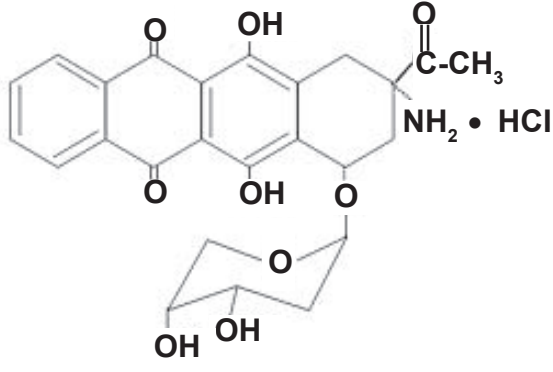

Amrubicin

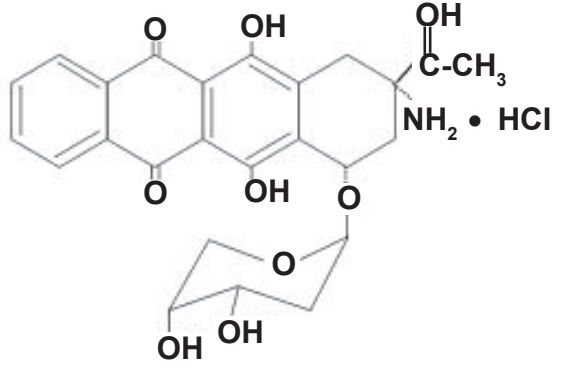

Amrubicinol

Figure I Structure of amrubicin and its active metabolite, amrubicinol.

\section{Preclinical studies}

In in vitro experiments, amrubicin and its metabolite amrubicinol have been found to be active against a broad spectrum of human cell lines established from cancers of the lung, prostate, urinary bladder, colon, kidney, pancreas, and uterus (Yamaoka et al 1998). Amrubicinol has been shown to exhibit a 20- to 220-fold more potent antitumor activity in vitro than amrubicin itself, with amrubicinol being as potent as doxorubicin. In addition, amrubicin and amrubicinol have also been demonstrated to show some degree of non-cross resistance with doxorubicin. However, like other anthracyclines, the cytotoxicity and intracellular accumulation of amrubicin in P-glycoprotein overexpressed MDR1 gene-transfected LLC-PK1 (L-MDR1) cells were significantly decreased compared with those in parental LLC-PK1 cells, suggesting that the antitumor efficacy of amrubicin is affected by the expression level of P-glycoprotein in lung cancer cells in chemotherapy (Takara et al 2002; Hira et al 2008).

Amrubicin has been shown to be more effective against 5 human xenografts (1 breast cancer, 1 lung cancer, and 3 gastric cancers), equally effective against 2 gastric cancers, and less effective against a lung cancer and 2 gastric cancer tumors, compared with doxorubicin (Morisada et al 1989; Noguchi et al 1998).
Amrubicin caused dose-dependent weight loss, ataxia, myelosuppression, and hair loss in mice after a single intravenous injection. The maximum tolerated dose (MTD) for such administration was estimated to be $25 \mathrm{mg} / \mathrm{kg}$ in 4 mouse strains.

Cardiotoxicity is one of the dose-limiting toxicities of anthracyclines. However, amrubicin showed little delayed-type cardiotoxicity in rabbit and dog experimental models (Suzuki et al 1997; Noda et al 1998). Furthermore, amrubicin did not aggravate doxorubicin-induced myocardial injury.

\section{Clinical studies}

\section{Amrubicin monotherapy}

Phase II study in previously untreated patients

with extensive-stage SCLC

Thirty-five previously untreated patients with extensive-disease SCLC (ED-SCLC) were enrolled in the study (Yana et al 2007). Amrubicin was administered as a daily intravenous injection of $45 \mathrm{mg} / \mathrm{m}^{2} /$ day for 3 consecutive days every 3 weeks. Of the 33 eligible patients, 3 showed a complete response (CR) and 22 showed a partial response (PR), with an overall response rate of $75.8 \%$ (95\% confidence intervals [CI], 57.7\%-88.9\%). 
The MST and 1-year survival periods were 11.7 months and $48.5 \%$, respectively. The most common toxicity was hematologic toxicity.

\section{Phase II trial for the treatment of refractory} or relapsed SCLC

SCLC patients with measurable disease who had been previously treated with at least one platinum-based chemotherapeutic regimen were entered into the trial (Onoda et al 2006). Since these patients had previously received chemotherapy or radiotherapy, the amrubicin was administered at a reduced dose of $40 \mathrm{mg} / \mathrm{m}^{2}$ per day $\times 3$ days every 3 weeks. Sixty patients ( 16 refractory and 44 sensitive) were enrolled. The overall response rates were 50\% (95\% CI, $25 \%-75 \%)$ in the refractory group and $52 \%(95 \% \mathrm{CI}$, $37 \%-68 \%)$ in the sensitive group. The overall survival and 1-year survival periods in the refractory-disease group were 10.3 months and $40 \%$, respectively, and in the sensitive-disease group 11.6 months and $46 \%$, respectively. The grade 3 or 4 hematologic toxicities were neutropenia $(83 \%)$, thrombocytopenia (20\%), and anemia (33\%). Febrile neutropenia was observed in 3 patients $(5 \%)$. The non-hematologic toxicities were mild. Thus, amrubicin showed significant activity against SCLC, with predictable and manageable toxicities.

\section{Randomized phase II trial for second-line treatment of sensitive SCLC}

A randomized phase II trial of amrubicin versus topotecan was conducted in patients with ED-SCLC sensitive to platinum-based first-line chemotherapy (recurrence or progression $\geq 90$ days after first-line chemotherapy (Jotte et al 2008). Patients were randomized at a $2: 1$ ratio to receive either amrubicin or topotecan. The interim data from 76 patients were as follows: amrubicin improved the overall response rate compared with topotecan ( $34 \%$ versus $3.8 \%$; $<<0.004$ ). A trend toward a longer progression-free survival time in the amrubicin group (4.6 months) compared with the topotecan group (3.5 months) was seen. They concluded that amrubicin is active in non-Japanese patients and is well tolerated, with myelosuppression being the main dose-limiting toxicity.

Phase II trial for second-line treatment of refractory SCLC

Patients with SCLC who progress while receiving first-line chemotherapy or who relapse within 3 months are much less likely to respond to additional chemotherapy and have an expected MST of 6 months or less. Single-agent topotecan in this population results in a response rate of $2 \%$ to $7 \%$.
Seventy-five patients were enrolled in a trial (Ettinger et al 2008). Of the 69 patients who were treated, 11 patients attained a PR and one patient had a $\mathrm{CR}$, with an overall response rate of $17.4 \%$, which compares favorably with the historically observed rate for topotecan. Thus, amrubicin is a promising new anthracycline warranting further study. A phase III trial in second-line SCLC patients is now underway, as well as a phase II study in first-line SCLC patients.

\section{Combination chemotherapy involving amrubicin}

Phase I-II study of amrubicin and cisplatin for previously untreated patients with extensive-stage SCLC

Patients with histologically or cytologically proven measurable ED-SCLC, no previous chemotherapy, and good prognostic factors were enrolled in this trial (Ohe et al 2005). Amrubicin was administered on days 1 to 3 and cisplatin was administered on day 1, every 3 weeks. Four patients were enrolled at dose level 1 (amrubicin, $40 \mathrm{mg} / \mathrm{m}^{2} /$ day and cisplatin, $60 \mathrm{mg} / \mathrm{m}^{2}$ ) and 3 patients were enrolled at dose level 2 (amrubicin, $45 \mathrm{mg} / \mathrm{m}^{2} /$ day and cisplatin $60, \mathrm{mg} / \mathrm{m}^{2}$ ). The maximum-tolerated dose and the recommended dose (RD) were found to be level 2 and level 1, respectively. The response rate at the RD was $87.8 \%(36 / 41)$. The median survival and the 1-year survival rate were encouraging, 13.6 months and $56.1 \%$, respectively.

\section{Discussion}

Because of the systemic nature of SCLC, chemotherapy offers the best hope of improving treatment outcomes. However, over the past 3 decades, therapeutic advances for this disease have occurred very slowly.

Amrubicin markedly inhibits topoisomerase II activity and has exhibited a broad spectrum of antitumor activity in preclinical studies (Yamaoka et al 1998). The results of clinical studies examining amrubicin as a single agent or as a component of combination regimens for SCLC have been promising, with manageable, non-cumulative toxicities (Ohe et al 2005; Onoda et al 2006; Yana et al 2007). At present, two randomized trials to determine the exact role of amrubicin in the treatment of SCLC are now underway in Japan: one examining cisplatin + amrubicin versus cisplatin + irinotecan in previously untreated patients with ED-SCLC, and one examining amrubicin versus carboplatin + etoposide in elderly patients with ED-SCLC. Two clinical trials have confirmed the effectiveness of amrubicin in US patients with SCLC. One of these trials was a randomized phase II trial comparing amrubicin with topotecan as a second-line treatment in a treatment 
sensitive-group (Ettinger et al 2008). The other was a phase II trial in patients refractory to first-line chemotherapy (Ettinger et al 2008). Amrubicin may be a promising new addition to treatment strategies for SCLC, especially for chemoresistant patients. However, there are only a few clinical trials of this agent and the number of the patients treated with this drug is still quite small. Therefore, further studies exploring the role of amrubicin as a single agent or in combination with cytotoxic agents as well as novel molecular-targeted drugs for the treatment of other various malignancies are warranted. Lastly, since most of the trials with amrubicin have been done in Japan, future clinical studies to define the benefits and risk associated with amrubicin as a single agent or in combination for cancer therapy conducted overseas are eagerly awaited.

\section{Disclosures}

The authors have no conflicts of interest to disclose.

\section{References}

Ettinger DS, Jotte RM, Gupta V, et al. 2008. A phase II trial of single-agent amrubicin (AMR) in patients with extensive disease small cell lung cancer (ED-SCLC) taht is refractory or progressive within 90 days of completion of first-line platinum-based chemotherapy. 44th Am Soc Clin Oncol Annual Meeting, Grunberg SM (ed), Vol. 26. pp. 434a: Chicago.

Hanada M, Mizuno S, Fukushima A, et al. 1998. A new antitumor agent amrubicin induces cell growth inhibition by stabilizing topoisomerase II-DNA complex. Jpn J Cancer Res, 89:1229-38.

Hira A, Watanabe H, Maeda Y, et al. 2008. Role of P-glycoprotein in accumulation and cytotoxicity of amrubicin and amrubicinol in MDR1 gene-transfected LLC-PK1 cells and human A549 lung adenocarcinoma cells. Biochem Pharmacol, 75:973-80.
Ishizumi K, Ohashi N, Tanno N, et al. 1987. Stereospecific total synthesis of 9-aminoanthracyclines: (+)-9-amino-9-deoxydaunomycin and related compounds. J Org Chem, 52:4477-85.

Jotte RM, Conkling PR, Reynolds C, et al. 2008. A randomized phase II trial of amrubicin (AMR) vs. topotecan as second-line treatment in extensivedisease small-cell lung cancer (SCLC) sensitive to platinum-based first-line chemotherapy. 44th Am Soc Clin Oncol Annual Meeting, Grunberg SM (ed), Vol. 26. pp. 433a: Chicago.

Morisada S, Yanagi Y, Noguchi T, et al. 1989. Antitumor activities of a novel 9-aminoanthracycline (SM-5887) against mouse experimental tumors and human tumor xenografts. Jpn J Cancer Res, 80:69-76.

Noda T, Watanabe T, Kohda A, et al. 1998. Chronic effects of a novel synthetic anthracycline derivative (SM-5887) on normal heart and doxorubicin-induced cardiomyopathy in beagle dogs. Invest New Drugs, 16:121-8.

Noguchi T, Ichii S, Morisada S, et al. 1998. In vivo efficacy and tumor-selective metabolism of amrubicin to its active metabolite. $J p n$ $J$ Cancer Res, 89:1055-60.

Ohe Y, Negoro S, Matsui K, et al. 2005. Phase I-II study of amrubicin and cisplatin in previously untreated patients with extensive-stage small-cell lung cancer. Ann Oncol, 16:430-6.

Onoda S, Masuda N, Seto T, et al. 2006. Phase II trial of amrubicin for treatment of refractory or relapsed small-cell lung cancer: Thoracic Oncology Research Group Study 0301. J Clin Oncol, 24:5448-53.

Sher T, Dy GK, Adjei AA. 2008. Small cell lung cancer. Mayo Clin Proc, 83:355-67.

Suzuki T, Minamide S, Iwasaki T, et al. 1997. Cardiotoxicity of a new anthracycline derivative (SM-5887) following intravenous administration to rabbits: comparative study with doxorubicin. Invest New Drugs, 15:219-25.

Takara K, Sakaeda T, Yagami T, et al. 2002. Cytotoxic effects of 27 anticancer drugs in HeLa and MDR1-overexpressing derivative cell lines. Biol Pharm Bull, 25:771-8.

Yamaoka T, Hanada M, Ichii S, et al. 1998. Cytotoxicity of amrubicin, a novel 9-aminoanthracycline, and its active metabolite amrubicinol on human tumor cells. Jpn J Cancer Res, 89:1067-73.

Yana T, Negoro S, Takada M, et al. 2007. Phase II study of amrubicin in previously untreated patients with extensive-disease small cell lung cancer: West Japan Thoracic Oncology Group (WJTOG) study. Invest New Drugs, 25:253-8. 